\title{
Immunotherapeutic approaches and vaccination strategies
}

Nowak, AK. MBBS FRACP. Medical Oncology Research Fellow. University Department of Medicine, University of Western Australia

Lake, RA. PhD. Research Fellow. University Department of Medicine, University of Western Australia

Robinson BWS. MD MBBS FRACP FACP FCCP DTM\&H MRCP. Professor of Medicine. University Department of Medicine, University of Western Australia 
Malignant mesothelioma is increasing in incidence and most commonly has a fatal outcome. Patients commonly present with advanced disease, making treatments such as surgery and radiotherapy anatomically and technically difficult and often unsuccessful in this group of patients. Furthermore, chemotherapy has historically been ineffective, although in recent years newer regimens with higher response rates have been reported (Byrne, Davidson et al. 1999; Nowak, Byrne et al. 2002; Vogelzang, Rusthoven et al. 2002), but are not curative. Hence there is a need for new treatments. New therapies such as immunotherapy and vaccine therapy can therefore be readily trialed in this disease. In this chapter, we will discuss the theoretical and preclinical bases of these experimental strategies, and provide an overview of the clinical trials performed in this area. Although this discussion separates immunotherapy, vaccine therapy, and combination chemo-immunotherapy, these areas are linked and such separations are artificial but necessary.

\section{The immune response to a solid tumor}

In order to understand how novel therapies may alter the immune response to tumor, it is important to first understand how the immune system responds to cancer under normal circumstances. The anti-tumor immune response consists of an innate or non-specific component and an adaptive or specific component. The innate and adaptive immune systems do not act in isolation, each producing soluble factors such as interferon-gamma (IFN- $\gamma$ ) and interleukins which may have a stimulatory or inhibitory role for cells from the other arm of the immune response, and there may also be cross-talk between cell populations from the innate and adaptive immune systems, adding further complexity to this interaction.

An effective immune response to tumor requires both recognition of tumor by the immune system and the subsequent development of an adequate immune response with the ability to infiltrate the tumor and kill tumor cells. The immune system must recognise tumor antigens, they must be taken up, processed, and presented to CD4+ T cells by dendritic cells, and directly or cross-presented to CD8+ T cells in the context of appropriate co-stimulation. CD8+ T cells must be capable of proliferating, entering the tumor milieu, and then effectively killing tumor or activating other local cells to do so. The magnitude and duration of the cellular response must be adequate to eradicate tumor. Important points in the development of anti-tumor immunity will be discussed. 


\section{The innate immune response}

The innate immune system originally evolved to produce a rapid, first-line defence against pathogens. It has not evolved to recognize antigens, and thus has a less important role in anti-tumor immunity than the adaptive immune system. However, NK cells may have an anti-tumor role as mediators of antibody dependent cellular cytotoxicity (ADCC), killing tumor cells coated with antibody via their receptor for IgG. Furthermore, they interact with the adaptive immune system by secreting IFN $\gamma$ and other cytokines such as interleukin 1 (IL-1) and GM-CSF when activated. They may also have a role in surveillance against malignant transformation. Neutrophils play an important role in acute inflammation and the immediate response against infection, but their role in anti-tumor immunity has received little attention, despite the fact that neutrophil infiltration of tumors corresponds with a favourable prognosis in some studies, and that most cytokine treatments of experimental tumors show a strong neutrophil infiltrate in responding sites (Di Carlo, Forni et al. 2001). Once recruited into the tumor, neutrophils can produce IL-1 $\beta$, tumor necrosis factor $\alpha$ (TNF- $\alpha$ ), and interferons, and can also kill tumor cells by ADCC (Kindzelskii and Petty 1999). Tumor macrophages infiltrates are often heavy, and these cells may have a role in antigen presentation and tumor cytotoxicity, eg. via production of NO.

\section{The adaptive immune response}

The adaptive immune response entails the interaction between $\mathrm{B}$ and $\mathrm{T}$ lymphocytes and antigens. The two arms of the adaptive immune system consist of the cellular or $\mathrm{T}$ lymphocyte arm, and the humoral or B lymphocyte/antibody arm. Although the adaptive immune system responds more slowly than the innate immune system, its advantage lies in its increased specificity. Whilst early stages of lymphocyte development are not dependent on the presence of antigen, their subsequent survival, differentiation and proliferation become antigen-dependent, underlying the specificity of adaptive immunity.

Tumor antigens are usually proteins, expressed by the tumor cell, which can be recognised by the adaptive immune system. They may result from somatic mutations in normal gene products, mutated oncogenes, viral proteins, normal gene products with a restricted tissue distribution such as the cancer-testis antigens, and normal tissue-specific gene products. It now seems clear that the failure to elicit an effective immune response is not due to a lack of tumor antigen expression, nor to lack of host recognition of tumor antigens. 
The failure to control or eradicate tumor appears to occur in the priming or licensing of the cell-mediated effector arm. Tumor antigens, including mesothelioma antigens, are constitutively presented in lymph nodes draining tumors and can stimulate a $\mathrm{T}$ cell proliferative response, regardless of where in the cell the tumor antigens are expressed (Robinson, Lake et al. 1999; Marzo, Lake et al. 1999a; Creaney, Mc Laren et al. 2001).

The cellular arm of the adaptive immune system is predominantly composed of $\mathrm{T}$ lymphocytes, and there is abundant evidence that these cells are important in anti-tumor immunity. CD4+ and CD8+ T lymphocytes can recognize antigen presented to them by antigen presenting cells (APCs) in the appropriate MHC context and with the correct expression of co-stimulatory molecules. Activated T cells can then proliferate and move from the lymph node to the periphery as functional immune effector cells. Both CD4+ and CD8+ $\mathrm{T}$ cells are important for optimal anti-tumor immunity, $\mathrm{T}$ cell subset depletion experiments showing that both cell types are required for rejection of tumor in vaccination studies (Pardoll and Topalian 1998). CD4+ lymphocytes have an important role at numerous points along the pathway of initiating, maintaining, and directing the activation of CD8+ $\mathrm{T}$ lymphocytes, but it is the CD8+ $\mathrm{T}$ cells that, when appropriately activated, differentiate to become cytotoxic effector cells, with the ability to target and kill tumor.

The humoral arm of the adaptive immune response consists of B lymphocytes and their differentiation into antibody-secreting mature plasma cells and antibodies. They may have a role as potential APCs, but they are not necessary for an efficient immune response, and may in some circumstances be detrimental to $\mathrm{T}$ cell activation and tumor recognition. Although antibody responses do not generally correlate with response against tumor, in some experimental situations, immune sera have been shown to enhance tumor growth, probably by blocking access of tumor specific lymphocytes to their target (Hellstrom and Hellstrom 1974). In addition, B cell-deficient mice in some systems control tumor growth more readily than their normal littermates, and the presence of B cells in the priming phase may result in disabled CD4+ T cell help for CD8 ${ }^{+}$CTL (Qin, Richter et al. 1998).

\section{How do tumors escape immune destruction?}

Early theories of anti-tumor immunity suggested that there was constant immune surveillance for tumors, which arose frequently but were then eliminated as tumor antigens were recognized as foreign or non-self. 
Tumors that did grow were felt to have escaped immune surveillance. However, this theory was challenged by the observation that immunodeficient mice did not have a higher incidence of tumor occurrence compared with immunocompetent mice, although recent data have again supported this notion. There are several explanations as to why the immune system fails to eradicate many tumors, even those expressing foreign antigens. Firstly, MHC class I molecule expression is necessary for antigen presentation to CD8+ T lymphocytes. Whilst most cells, including tumor cells, express MHC class I molecules, some tumors can downregulate class I expression. The progressive loss of class I antigens may have a role in tumor progression and the development of metastatic potential. This supports a "surveillance" mechanism because selection pressure by CD8+ T cells must be present for class I loss variants to occur. Secondly, even when tumor cells express MHC class I molecules, they do not generally express co-stimulatory molecules of the B7 family and are therefore unable to provide signal two, which may lead to anergy and consequent lack of proliferation and interleukin-2 (IL-2) production if the CD8+ T cell TCR becomes engaged (DeSilva, Urdahl et al. 1991). Thirdly, even if CD8+ lymphocytes are appropriately stimulated, the tumor may provide an inhospitable environment for effector lymphocytes. They may be large and poorly vascularised, and the endothelium and extracellular matrix may prevent $\mathrm{T}$ cell infiltration. The tumor environment can contain numerous immunosuppressive cytokines, including TGF- $\beta$, IL-10, and vascular endothelial growth factor (VEGF) which may enhance the ability of the tumor to evade host immune responses. The expression of Fas ligand on tumor cells may induce tumor-reactive CTLs to enter the apoptotic pathway on contact with tumor. Finally, even if tumors stimulate an effective immune response, they may be too large for effective immunological destruction when this response matures. It is possible that tumor must reach a certain critical mass to activate naïve cells, trigger expansion, and induce effector function. They may be ignored early in their development because antigen presentation levels remain below a certain threshold. Necrosis and apoptosis are more likely to occur in larger tumors, with tissue damage activating host DCs to acquire antigen and present it to tumor specific T-cells in the draining lymph node. However by the time an effective anti-tumor response has been stimulated it may be too late. It is likely that some or all of these factors contribute in varying degrees to the ability of individual tumors to escape immune destruction, and the problem is almost certainly multifactorial. 


\section{Immunotherapy in malignant mesothelioma}

Why could immunotherapy be effective in malignant mesothelioma?

Although malignant mesothelioma does not belong to the group of classically immunogenic tumors such as malignant melanoma and renal cell carcinoma, in which spontaneous regression is sometimes reported, there is good evidence that the immune system can recognise this tumor. There is a relationship between tumor infiltrating lymphocytes and prognosis, which suggests that improving the immune response to this tumor may improve patient outcome (Leigh and Webster 1982). Furthermore, spontaneous regression has been reported in this disease, associated with evidence of immune responsiveness (Robinson, Robinson et al. 2001) (Figure 1). The impetus to pursue immunotherapy in mesothelioma arose because of a lack of effective conventional therapies, coupled with the fact that mesothelioma patients, due to their otherwise poor prognosis, are often motivated and willing participants in clinical trial programs. As mesothelioma is often localised to the pleural space, which is readily accessible, local treatments can be trialed. Similarly, it is not difficult to obtain tumor tissue to study surrogate biomarkers of treatment efficacy such as expression of transduced genes and lymphocytic infiltration of tumor. Further support for this treatment strategy comes from evidence that both mesothelioma patients and asbestos exposed persons without mesothelioma have some immune dysfunction, and in vitro studies also support the detrimental effects of asbestos on immune cells. Although patients with mesothelioma maintain normal white cell counts, immunoglobulin levels and total serum proteins, they show decreased mitogen responsiveness and lymphokine-activated killer (LAK) cell activity against mesothelioma tumor targets (Haslam, Lukoszek et al. 1978; Manning, Davis et al. 1991; Garlepp, D et al. 1992). Both humoral and cell-mediated antibody-dependent cellular toxicity is abnormal (Lew, Tsang et al. 1986); natural killer (NK) cell activity is decreased, and CD4+ lymphocyte numbers are reduced. T cells infiltrating murine mesotheliomas also show low surface expression of CD3, a feature of $\mathrm{T}$ cell down-regulation. Furthermore, asbestos itself suppresses NK and LAK cell function in vitro (Robinson 1989; Manning, Davis et al. 1991; Bielefeldt-Ohmann, Jarnicki et al. 1996). Thus, it may be reasonable to explore treatments which can improve the immune response to this disease. 


\section{Tumor antigens in malignant mesothelioma}

In order for the immune system to mount a response against tumor, the tumor must express antigens which distinguish them from the surrounding normal tissue. Nevertheless, despite the presence and effective presentation of antigens, many tumors still evade immunological destruction. Thoracic malignancies, including mesothelioma, have long been considered non-immunogenic and not amenable to immunotherapy. However, the lack of an effective immune response is unlikely to be due to lack of antigen expression, with several tumor antigens being recently identified in malignant mesothelioma. The cancer-testis antigens are normal gene products, but usually have a restricted tissue distribution. These include the MAGE and GAGE antigen groups. Malignant mesothelioma cells have been recently found to express numerous cancer-testis antigens, with individual cells expressing up to four concurrently. These included MAGE-1, -2, and -3, GAGE 1-2, GAGE 1-6, SSX-2, and SSX 1-5 (Sigalotti, Coral et al. 2002). The SEREX technique (serologic analysis of recombinant complementary DNA (cDNA) expression libraries) identifies anti-tumor antibody responses by using sera from the patients to probe tumor cDNA libraries and identify antigens generating a humoral response. Antigens discovered by SEREX techniques generally also elicit a cellular response (Jager, Chen et al. 1998), and the majority of tumor antigens recognized by this analysis are self antigens, suggesting that reactions occurring to immunogenic proteins to which the host is not fully tolerant, as a form of auto-immunity (Naftzger, Takechi et al. 1996). We have identified several tumor antigens in mesothelioma using SEREX (Robinson, Callow et al. 2000). Antibodies to topoisomerase IIb were found in 13 of 14 patients, and thus there may be shared tumor

antigens in mesothelioma. Although none of the antibodies found in this study were unique to mesothelioma, this finding merely supports the idea that immunogenic tumors more commonly overexpress self-antigens rather than novel tumor antigens. As mesotheliomas express tumor antigens, and these antigens can be recognized by the host, antigen expression and recognition are unlikely to be the limiting factors in the anti-tumor immune response.

\section{Antigen presentation and co-stimulation}

Tumor antigens are presented to CD8+ T cells in the context of the class I MHC molecule. The expression of MHC antigens by tumors may affect antigen presentation to CD8+ T cells and APC's, and the influence 
the subsequent immune response. Although human malignant mesothelioma cell lines express high levels of class I MHC molecules, the tumor cells are poor APCs (Christmas, Manning et al. 1991). APCs such as dendritic cells (DCs) upregulate their expression of co-stimulatory molecules like B7-1 and MHC class II molecules after receiving signals from chemokines and cytokines (GM-CSF, IL-4, TNF $\alpha$, IL-1 $\beta$ ) and bacterial products (lipopolysaccharide, LPS). Final maturation can then occur after DCs interact with CD4+ T cell surface molecules (CD40-CD40 ligand interaction) and cytokines such as IFN $\gamma$, and further co-stimulatory molecules are expressed. Whilst tumors express MHC class I molecules, they rarely express co-stimulatory molecules or secrete cytokines, and thus cannot present antigen in the right context to stimulate a cytotoxic response. The provision of co-stimulation by transfecting B7-1 into tumor has been trialed in mesothelioma animal models. Transfecting B7-1 into non-immunogenic mesothelioma cell lines inhibited tumor growth, and mice also rejected the parent cell line, although rejection was not CD4+ independent. Cytotoxic T cells were effectively generated. In the same system, transfection of tumor with B7-2 did not affect the development of tumors in vivo (Leong, Marley et al. 1997). The murine mesothelioma cell lines used in these experiments were asbestos-induced and are similar in many respects to human malignant mesothelioma (Davis, Manning et al. 1992). These results suggest that transfecting tumor cells with B7 may allow them to present antigen better to effector T cells, probably by increasing antigen delivery via host APCs. This approach could be used clinically in cancer vaccination by transfecting allogeneic tumors. There have been no clinical trials of this approach as yet in mesothelioma.

\section{Non-specific immunostimulants}

The history of immunotherapy in mesothelioma mirrors its development in other tumor types. In the 1970s, bacillus Calmette-Guerin (BCG) was widely tested as an immunotherapy in many tumor types, including mesothelioma (Webster, Cochrane et al. 1982). Later, NK cells were discovered, and interleukins which could stimulate the development of LAK cells became available as purified recombinants. In the following decade, NK and LAK cell therapy, together with cytokine therapy became a major focus of research in many malignancies. NK cells are cytotoxic to both virally infected cells and tumor cells. They are able to mediate direct lytic effects on virally infected cells and can also mediate antibody dependent cellular cytotoxicity (ADCC). LAK cells are generated by culturing leukocytes together with high doses of IL-2 for 
several days, and IL-2 and the interferons increase the cytotoxicity of this population. They can lyse not only those cells which are susceptible to NK cell lysis, but also fresh tumor cells which are normally resistant to NK lysis (Grimm, Mazumder et al. 1982). Both fresh mesothelioma cells and cultured cell lines are sensitive to lysis by IL-2 activated LAK cells (Manning, Bowman et al. 1989) although they are not destroyed by NK cells. Despite these interesting pre-clinical observations, LAK cell therapy was toxic in clinical practice and trialed only in small numbers of patients with mesothelioma.

\section{Cytokine therapy}

Since the large scale production of cytokines and interferons became possible, interest in the therapeutic possibilities of these proteins has risen. Following antigen presentation, the cytokine environment is responsible for promoting an optimal Th1 type response or, instead, a less effective Th2 type humoral response. A Th1 response produces IL-2, TNF $\alpha$ and IFN $\gamma$, which mediate local inflammation and cytotoxicity, inducing a strong cellular immune response. A Th2 response produces IL-4, IL-5, IL-6, and IL-10, which stimulate B cell proliferation and subsequent antibody production. Hence, if the tumor cytokine milieu can be changed to promote a Th1 response, anti-tumor immunity may be generated more effectively. In mesothelioma, the pleural space is accessible to treatment strategies attempting to instill or induce high cytokine levels in the local tumor area, and this tumor rarely metastasizes. Hence, this approach has been exploited in both animal models and patients. A further benefit of intrapleural cytokine administration in this disease is the decrease in systemic side effects of cytokines, which can be severe and even cause mortality. High intratumoral cytokine concentrations can be achieved by either transducing tumor cells to express high cytokine levels using a viral vector (Mukherjee, Haenel et al. 2000) or by injecting or infusing the cytokine directly into the tumor (Davidson, Musk et al. 1998). The first strategy will be discussed later with other methods of gene therapy.

The interferons are cytokines with antiviral activity which have both direct antiproliferative effects and a role in regulation of gene expression. Interferon alpha (IFN $\alpha$ ) induces increased cell surface expression of MHC molecules on both normal and malignant cells, which may increase the susceptibility of malignant cells to immune destruction. Pre-clinically, the effects of IFN $\alpha$ on malignant mesothelioma cell lines are 
variable (Bowman, Manning et al. 1991; Hand, Husgafvel-Pursiainen et al. 1991), and both human and murine malignant mesothelioma cell lines can be inhibited by IFN $\alpha$ in animal models, independent of the presence of T cells (Suzuki, Chahinian et al. 1987; Bielefeldt-Ohmann, Fitzpatrick et al. 1995). Following these pre-clinical observations, IFN $\alpha$ was trialed in patients, but has not fulfilled its promise. One of thirteen patients treated with systemic IFN $\alpha$ showed an objective tumor response (Ardizzoni, Pennucci et al. 1994), and intracavitary treatment for malignant pleural effusion from mesothelioma did not give any objective responses (Lissoni, Barni et al. 1992). In our centre, a response rate of 13\% was observed from 25 patients treated with systemic therapy (Christmas, Manning et al. 1993). Although IFN $\alpha$ shows additive or synergistic tumor inhibition both in vitro and in vivo when combined with TNF $\alpha$ (Hand, HusgafvelPursiainen et al. 1991) IFN $\gamma$, and the cytotoxic drug methotrexate (Hand, Pelin et al. 1995), this approach has been difficult in the clinical situation. A combination of doxorubicin and IFN $\alpha$ achieved a $16 \%$ partial response (PR) rate, but was too toxic for further development (Upham, Musk et al. 1993).

IFN $\gamma$ also shows anti-proliferative activity in vitro, alone and in combination with TNF $\alpha$ (Bowman, Manning et al. 1991; Hand, Husgafvel-Pursiainen et al. 1991). In vivo effects may be partially mediated through upregulation of MHC expression on mesothelioma cells, improving antigen presentation and hence acting as a better target for lytic T cells (Christmas, Manning et al. 1991). Whilst intrapleural IFN $\gamma$ has been trialed in the phase II setting, it has only shown any significant efficacy in early stage disease. Of 12 patients with stage I disease, there were four complete and one partial response. However, only one of ten patients with stage II disease attained a partial response (Boutin, Viallat et al. 1991). The combination of IFN $\gamma$ and TNF $\alpha$ was toxic in a phase II study of patients with solid tumors, although one patient with mesothelioma was noted to clear his ascites of malignant cells (Smith, Urba et al. 1991). Interferon beta (IFN $\beta$ ) also inhibits human mesothelioma cell growth in vitro (Von Hoff and Huong 1988), however it has not produced any responses in patients (Von Hoff, Metch et al. 1990), and was highly toxic. Single-agent interferons have not been developed further for mesothelioma, in part due to their high toxicity. All patients develop fever, and intrapleural therapy has been complicated by empyema. 
IL-2 is an autocrine T-cell growth factor released by these cells after antigen recognition. It is required for survival and proliferation of activated T-cells, and production is augmented by CD28 co-stimulation. Without IL-2 and co-stimulation, the T cell may become anergic or die. In addition, in some situations IL-2 can have a direct anti-proliferative effect on human malignant mesothelioma cells in vitro, independent of its effects on the cellular immune response (Porta, Danova et al. 2000). The ability of IL-2 to induce activation and expansion of LAK cells led to interest in clinical trials of this cytokine. Whilst in vivo murine mesothelioma growth is inhibited by combination therapy using IL-2 and LAK cells (Manning et al, unpublished data), no responses were seen in a pilot study of intrapleural IL-2/LAK cells in 5 patients with this disease, and the toxicity of the combination was excessive, even when IL-2 was given intrapleurally (Robinson, Bowman et al. 1993). There have been several further clinical trials of IL-2 in mesothelioma. A five-day continuous infusion of intrapleural IL-2 was reported to give an objective response in seven of 15 patients in a phase I study (Astoul, Viallat et al. 1993), and a follow-up to this study, giving $21 \times 10^{6} \mathrm{IU}$ IL-2 daily for 5 days reported 12 objective responses in 22 patients (54\%) and a survival benefit in responding patients (28 months vs. 8 months) (Astoul, Picat-Joossen et al. 1999). Despite this short, high dose course, little toxicity was reported. Low dose, daily intrapleural administration of IL-2 (14 days in a 4-week cycle) was not as effective, with 4 of 21 evaluable patients with stage I/II disease achieving a partial response (Goey, Eggermont et al. 1995). Intrapleural IL-2 levels were up to 6000 fold greater than systemic levels in this trial, and LAK activity was seen in intrapleural mononuclear cells from all patients. The dose was limited by fever, flu-like symptoms, and catheter infection.

Another interleukin, interleukin-12 (IL-12) has also been studied in murine and human mesothelioma. Systemic IL-12 could prevent tumor growth in some mice in a murine mesothelioma model, and tumor regression and growth inhibition occurred following intralesional IL-12 injection. This correlated with an increased intratumoral infiltrate of CD4+ and CD8+ T-cells (Caminschi, Venetsanakos et al. 1998). When a murine mesothelioma cell line was transfected with the IL-12 gene, tumor did not grow, and furthermore, paracrine secretion of IL-12 slowed growth of distally implanted tumor. IL-12 has not been tested clinically in this disease. 
Granulocyte-macrophage colony stimulating factor (GM-CSF) has numerous actions which may be beneficial to anti-tumor immunity, including increasing lymphocyte activation, augmenting antigen presentation, stimulating antigen presentation by dendritic cells and macrophages, and enhancing monocyte anti-tumor activity in vitro. GM-CSF has been transduced into murine tumors, and can give durable, specific anti-tumor immunity (Colombo, Ferrari et al. 1991; Dranoff, Jaffee et al. 1993). In a murine mesothelioma model, transfection of GM-CSF gives a dose-dependent anti-tumor effect (Davidson JA et al. Unpublished observations). Clinically, 6 patients with stage II disease received intralesional rhGM-CSF infused for 8 weeks via catheter in a pilot clinical trial (Davidson, Musk et al. 1998). A lymphocytic tumor infiltrate was seen in one patient who had a partial response, and tumor necrosis occurred in one patient, but only at the catheter site. The technical demands and toxicity of this approach precluded further study.

\section{Immunomodulatory gene therapy}

As an alternative to giving local or systemic treatment with cytokines, tumors can be transfected with genes for cytokines to induce anti-tumor immunity through paracrine secretion. Tumors producing IL-2 are heavily infiltrated by lymphocytic cells, whereas IL-4 producing tumors stimulate a massive infiltrate of macrophages and eosinophils but few T cells (Tepper, Pattengale et al. 1989; Tepper, Coffman et al. 1992; Pardoll 1995). Hence, genes for both these cytokines have been transfected into tumors in an attempt to activate CD4+ T cells or give direct stimulation to CD8+ T cells (reviewed in Parmiani, Colombo et al. 1997). In animal models, transfection of IL-4 into a tumor cell line both mediates rejection of the transfected cell line, and can generate immunity sufficient to reject the untransfected parent cell line, a phenomenon primarily mediated by CD8+ T cells (Golumbek, Lazenby et al. 1991). Tumor cells transfected to secrete IL-2 also produced an anti-tumor response protecting against subsequent challenge with the parental cell line, an effect not requiring CD4+ T cells (Fearon, Pardoll et al. 1990). Other cytokines which have been similarly trialed in vivo include IFN $\gamma$, IFN $\alpha$, TNF $\alpha$, GM-CSF, IL-6 and IL-1 $\beta$ (Gansbacher, Bannerji et al. 1990; reviewed in Parmiani, Colombo et al. 1997). Our group has transfected a series of cytokine genes into malignant mesothelioma cell lines. Tumors transfected with IL-2, IL-4, IL-12 or GM-CSF were all rejected in vivo in a murine mesothelioma model (Leong, Marley et al. 1997; Caminschi, Venetsanakos et al. 1999). Although all transfected cell lines were rejected, rejection of the 
parental cell line was not seen for any of these cell lines (Caminschi, Venetsanakos et al. 1999), suggesting that the clinical utility of this approach may be limited. However, the combination of debulking surgery and cytokine gene therapy can reduce the rate of tumor growth in an experimental untransfected metastasis (Mukherjee, Nelson et al. 2001). Those transfectants expressing B7.1 or producing high GM-CSF levels were most effective at reducing tumor growth rate, whilst those producing IL-2 and IL-4 did not show much growth delay. This combination of debulking surgery and cytokine gene therapy has not yet been trialed clinically.

In patients, gene therapy has been explored as a way of delivering cytokines in order to circumvent some of the problems of infusional cytokine treatment: it may enable the cytokine to be continuously produced, in high levels, and in the correct location. In reality, however, the ideal vector to fulfil this promise has not yet been identified. Our group has studied vaccinia virus (VV) as a vector for cytokine gene transfer in malignant mesothelioma (Mukherjee, Haenel et al. 2000). IL-2 gene was inserted into the TK region of the vaccinia virus, which then became replication deficient. Six patients received one to three weekly intratumoral injections of vaccinia virus-IL-2 (VV-IL-2), receiving up to $10^{7}$ plaque forming units (PFU) per injection. Previous studies suggested that vaccinia virus was immunogenic and hence unlikely to maintain long-term expression of the transfected cytokine. VV IgG titres did increase over the treatment course; however, tumor biopsies showed prolonged, although low-level intratumoral expression of VV-IL2 mRNA. Virus was neither excreted nor transmitted to contacts. Although there were no tumor responses, $50 \%$ of injection-site biopsies showed $\mathrm{T}$ cell infiltration. We are continuing to investigate cytokine gene transfection in animal models of mesothelioma. Nevertheless, it is debatable whether continuous cytokine production, as occurs with successful delivery of gene therapy, is the ideal schedule for cytokine administration. Data from animal models has shown that continuous infusion of IL2 is less effective in producing tumor remission than intermittent injections (van Bruggen et al. Unpublished data).

\section{Mesothelioma vaccines}

Cancer vaccines aim to stimulate a specific immune response in the tumor host. For a vaccine to have any degree of success, the patient must have an intact cell-mediated immune system, and the tumor must bear antigen/s which are recognizable and can generate an immune response. This therapeutic strategy is most 
likely to succeed where the tumor burden is low (adjuvant therapy following chemotherapy or surgery) or as a low-toxicity prophylaxis in high risk populations, such as those exposed to high levels of asbestos. The tumor antigen does not need to be identified in order to use vaccination as a treatment strategy. When the tumor does not express known antigens, autologous whole tumor cells or tumor lysates may be used or, less attractive, allogeneic cells. Production of tumor lysates can expose intracellular proteins, which may encourage a more immunogenic mode of presentation. Autologous tumor can be surgically obtained and then inactivated by enzymatic digestion, freezing, thawing, and then irradiation. Allogeneic tumor can be obtained from one or several cell lines which can be mixed and irradiated. The success of allogeneic tumor vaccines requires shared tumor antigens between the autologous tumor and the patient's tumor. If shared tumor antigens have already been identified, antigenic peptides or proteins can be manufactured, or APCs transfected with genes for tumor antigens. An example of the identification and use of a possible shared tumor antigen as a vaccine in mesothelioma is the use of vaccinia virus transfected with a modified SV40 T antigen (Imperiale, Pass et al. 2001). This vaccine has been tested in animal models, and found effective against tumor challenge and also established tumors. It has not been tested clinically in this disease.

The availability of the right antigen is important, but without the appropriate context of antigen presentation, anergy, ignorance, or clonal deletion may occur in the specific T cell population. The "Danger Theory” suggests that signals from inflammation or tissue destruction may be necessary for appropriate antigen presentation (Matzinger 1998). The absence of "danger” may be why some antigen-bearing tumors do not stimulate an immune response. A successful vaccine must be able to either break tolerance, or activate $\mathrm{T}$ cells of different specificities, such as to low affinity or cryptic antigens. One method of increasing the immunogenicity of vaccines is to use an "adjuvant". These may be non-specific (BCG, Detox (Ribi Immunochem Research, Hamilton, MT), or the glycoside QS-21). LPS and CpGs, which bind toll-like receptors, are also excellent adjuvants (Medzhitov and Janeway 2000). Immunostimulatory cytokines such as GM-CSF are effective adjuvants (Simons, Mikhak et al. 1999), and the genes for GMCSF or other cytokines and co-stimulatory molecules can be transduced into tumor cells prior to vaccination (Pardoll 1995), although this approach is not really technically feasible for autologous vaccination. Autologous tumor may be more readily mixed with microspheres containing cytokines or with inert bystander cells previously transduced with the gene of interest (Pardoll 1998). In murine models, 
tumors transfected with the gene for GM-CSF can protect from tumor development and further challenge with the untransfected parent cell line (Dranoff, Jaffee et al. 1993). The use of adjuvant GM-CSF been trialed clinically in colon and prostate cancer and melanoma. We are currently conducting a pilot study of autologous vaccination in malignant mesothelioma. Tumor is removed by resection or thoracoscopically, then inactivated by freeze-thawing and subsequent irradiation. Tumor cells are mixed with soluble GMCSF, and injected subcutaneously, fortnightly for three months. There is an initial lead-in period of nine weeks in order to monitor baseline anti-tumor immune responses. Although tumor response and survival will be monitored, the primary aim of this pilot study is to demonstrate an immune response. This is assessed using western blotting and ELISA of serial serum samples, together with DTH skin testing using lysed irradiated autologous tumor cells. To date, eight patients have completed the trial. Two patients had a positive initial DTH skin test, and two further patients have subsequently developed positive DTH tests. One patient had a positive initial western blot, and one patient has developed a positive western blot after vaccination. Toxicity has been acceptable, with no hospital admission related to vaccination treatment. Median survival from diagnosis to date is 12 months, with four of eight patients remaining alive. The longest surviving patient is alive 25 months post diagnosis, and this patient had an initial positive DTH skin test which has remained positive throughout the vaccination programme. The development of tumorspecific immunity in some patients is encouraging, and the trial is ongoing.

\section{Tumor vaccine with suicide gene therapy}

A further approach currently under investigation is the use of allogeneic irradiated ovarian cancer cells which have been transduced with the gene for HSV-tk (PA-1-STK cells). "Vaccination” with these cells is followed by systemic treatment with ganciclovir. In vitro, these cells are capable of killing both murine and human mesothelioma cell lines, and when used in vivo, improved survival in a murine model of mesothelioma (Schwarzenberger, Lei et al. 1998). In human trials, radiolabelled PA-1-STK cells have been instilled into the pleural cavity of mesothelioma patients, and can traffic to intrapleural tumor (Harrison, Schwarzenberger et al. 2000). This system is now being trialed in a phase I setting in conjunction with ganciclovir treatment. It is hoped that a "bystander" effect will kill neighboring mesothelioma cells (Schwarzenberger, Harrison et al. 1998). The concomitant production of pro- 
inflammatory cytokines such as IL-2 and TNF $\alpha$ may also induce a more immunostimulatory tumor microenvironment (Schwarzenberger, Harrison et al. 1998). A clinical trial has been reported, with six patients treated with up to $1 \times 10^{8}$ PA1-STK cells alone and a further three patients receiving intrapleural PA1-STK cells followed by ganciclovir treatment. In results reported to date, increased CD8 T lymphocytes have been shown to enter the pleural fluid after this treatment, and no alloreactivity has been reported (Schwarzenberger, Harrison et al. 1998).

\section{Chemo-immunotherapy}

Until recently, there has been no "gold standard" for chemotherapy in the treatment of mesothelioma. This has opened possibilities for investigational treatments combining chemotherapy and immunotherapy. Much of this work has been trialed directly in patients with little pre-clinical exploration of the combination in models of mesothelioma. It has been hypothesized that the inflammation induced by an antecedent tumor vaccine may disrupt tumor architecture, thus possibly increasing the effect of cytotoxic drugs. However, there have also been theoretical concerns that chemotherapy may be detrimental to antigen-specific antitumor immunity, due its toxic effects on dividing lymphocytes.

We have been examining the effects of chemotherapy on antigen-specific anti-tumor immunity and combination chemo-immunotherapy in a murine model of mesothelioma. The cell line used was generated from asbestos inoculation into the peritoneal cavity of mice, and has subsequently been transfected with the hemagglutinin antigen (HA) as a tumor "neo-antigen” (Marzo, Lake et al. 1999b). The immune response can then be followed in vivo using, amongst other tools, T cell receptor transgenic mice with CD4+ and CD8+ $\mathrm{T}$ cells with specificity for this antigen. We have shown that gemcitabine chemotherapy decreases humoral immunity and inhibits B cell proliferation (Nowak, Robinson et al. 2002). However, gemcitabine appears to have a priming effect on cell-mediated immunity, increasing cross-presentation, $\mathrm{T}$ cell proliferation to specific tumor antigens, and tumor lymphocyte infiltration (Nowak et al. Manuscript submitted). Immunotherapy with a virus expressing the HA antigen is more effective when given after a full course of gemcitabine chemotherapy than when given alone. Furthermore, treatment of established 
murine mesothelioma using gemcitabine followed by immunotherapy with an activating anti-CD40 antibody results in cures of between 40 and $80 \%$ of mice. Alternative schedules using immunotherapy prior to or during chemotherapy are not effective (Nowak et al, manuscript in preparation). CD40 activation alone has not been trialed in mesothelioma, however this strategy was ineffective in clinical trials in other cancers (Vonderheide, Dutcher et al. 2001) as well as alone in murine mesothelioma (Nowak et al, manuscript in preparation). Nevertheless, it would be interesting to perform a clinical trial of this combination in mesothelioma, particularly in a setting of minimal residual disease, such as the post-surgical period.

In clinical trials, combining chemotherapy with cytokines has been most widely explored. A trial showing some minor activity of IFN $\alpha$ alone (Christmas, Manning et al. 1993) was followed by a trial of IFN $\alpha$ together with doxorubicin (Upham, Musk et al. 1993). Four of 25 patients treated (16\%) had a partial response to treatment, a response rate not different to either agent alone. However, the regimen was unacceptably toxic. IFN $\alpha$ was also combined with mitomycin C and cisplatin, following encouraging preclinical data (Sklarin, Chahinian et al. 1988; Metintas, Ozdemir et al. 1999). This was a non-randomised two-arm study, comparing 28 day cycles of IV cisplatin and mitomycin and subcutaneous IFN $\alpha$ with a group receiving best supportive care. There was no difference in survival between the two groups, and six of 43 patients (14\%) had a partial or complete response. IFN $\alpha$ and cisplatin have been trialed together, in a combination which was toxic but gave partial responses in 10 of 25 patients (40\%) (Soulie, Ruffie et al. 1996). A subsequent study by the same investigators gave a $50 \%$ higher dose of IFN $\alpha$, which was not more efficacious (PR 26\%) and was too toxic to pursue further (Trandafir, Ruffie et al. 1997). The response rate was not further improved by the addition of mitomycin C or IL-2 (Fizazi, Caliandro et al. 2000). The group performing these trials has now abandoned this approach in mesothelioma in favour of combination raltitrexed and oxaliplatin. Finally, IFN $\alpha$ and carboplatin have been trialed together with a partial response rate of 7\%, no different to carboplatin alone (O'Reilly, Ilson et al. 1999).

A recent trial of chemo-immunotherapy has used a different approach. SRL172 is a suspension of heatkilled mycobacterium vaccae, which has been used in combination with mitomycin C, vinblastine, and cisplatin (O'Brien, Saini et al. 2000). Theoretically, when chemotherapy kills tumor cells, tumor-specific or 
tumor-associated antigens may be released and this chemotherapy effect, in combination with the nonspecific stimulant of SRL172, could lead to improved antigen recognition. Nine trial patients had mesothelioma, and of these, two of four patients in the combination therapy group had a partial response, compared with one of five in the chemotherapy-only group. Newer, more active chemotherapy combinations, together with appropriate scheduling of chemotherapy and immunotherapy on the basis of pre-clinical information, should lead to improved efficacy of chemo-immunotherapy combinations.

\section{Conclusions and Future directions}

There has been much recent progress in the understanding of the immunology of malignant mesothelioma, particularly in identification of shared tumor antigens and in tumor models of the disease expressing neoantigens. However, gene therapy, immunotherapy, and cancer vaccination are yet to be established as conventional therapies. As more effective chemotherapy is becoming available (Byrne, Davidson et al. 1999; Nowak, Byrne et al. 2002; Vogelzang, Rusthoven et al. 2002), we may no longer be able to treat patients on clinical trials of immunotherapies as first-line treatment, although as yet there is no evidence that early chemotherapy is more beneficial for survival than delayed chemotherapy. Patients will be presenting for experimental therapies in the context of chemotherapy failure, together with chemotherapy, or in a post-surgical setting. Traditional markers of response may be obscured, emphasising the need to develop alternative biomarkers of efficacy which can be used at an earlier stage of disease. Techniques such as microarray may help us to predict which patients are likely to respond to treatments such as chemotherapy or immunotherapy. The principles of advances in immunotherapy in other malignancies can be applied to mesothelioma, at least to generate hypotheses and suggest new therapies for trial in animal models. It seems clear that single modalities of conventional therapies will not be successful alone, and we must look at combinations of treatments which include novel agents. 


\section{Acknowlegments}

The authors would like to thank our colleagues in the Tumor Immunology Group, University Department of Medicine, University of Western Australia for providing us with their unpublished data. AKN is the recipient of an Eva KA Nelson scholarship from the University of Western Australia. 
Ardizzoni, A., M. C. Pennucci, B. Castagneto, G. L. Mariani, A. Cinquegrana, D. Magri, A. Verna, F. Salvati and R. Rosso (1994). "Recombinant interferon alpha-2b in the treatment of diffuse malignant pleural mesothelioma." American Journal of Clinical Oncology 17(1): 80-82.

Astoul, P., D. Picat-Joossen, J. R. Viallat and C. Boutin (1999). "Intrapleural administration of interleukin2 for the treatment of patients with malignant pleural mesothelioma: a Phase II study." Cancer 83(10): 2099-2104.

Astoul, P., J. R. Viallat, J. C. Laurent, M. Brandely and C. Boutin (1993). "Intrapleural recombinant IL-2 in passive immunotherapy for malignant pleural effusion." Chest 103(1): 209-213.

Bielefeldt-Ohmann, H., D. R. Fitzpatrick, A. L. Marzo, A. G. Jarnicki, A. W. Musk and B. W. Robinson (1995). "Potential for interferon-alpha-based therapy in mesothelioma: assessment in a murine model." J.Interferon.Cytokine.Res. 15(3): 213-223.

Bielefeldt-Ohmann, H., A. G. Jarnicki and D. R. Fitzpatrick (1996). "Molecular pathobiology and immunology of malignant mesothelioma." Journal of Pathology 178(4): 369-378.

Boutin, C., J. R. Viallat, Z. N. Van, J. T. Douillard, J. C. Paillard, J. C. Guerin, P. Mignot, J. Migueres, F. Varlet and A. Jehan (1991). "Activity of intrapleural recombinant gamma-interferon in malignant mesothelioma." Cancer 67(8): 2033-2037.

Bowman, R. V., L. S. Manning, M. R. Davis and B. W. Robinson (1991). "Chemosensitivity and cytokine sensitivity of malignant mesothelioma." Cancer Chemother.Pharmacol. 28(6): 420-426.

Byrne, M. J., J. A. Davidson, A. W. Musk, J. Dewar, G. van Hazel, M. Buck, N. H. de Klerk and B. W. Robinson (1999). "Cisplatin and gemcitabine treatment for malignant mesothelioma: a phase II study." Journal of Clinical Oncology. 17(1): 25-30.

Caminschi, I., E. Venetsanakos, C. C. Leong, M. J. Garlepp, B. W. Robinson and B. Scott (1999). "Cytokine gene therapy of mesothelioma. Immune and antitumor effects of transfected interleukin-12." American Journal of Respiratory Cell \& Molecular Biology 21(3): 347-356.

Caminschi, I., E. Venetsanakos, C. C. Leong, M. J. Garlepp, B. Scott and B. W. Robinson (1998). "Interleukin-12 induces an effective antitumor response in malignant mesothelioma." Am.J.Respir.Cell Mol.Biol. 19(5): 738-746.

Christmas, T. I., L. S. Manning, M. R. Davis, B. W. Robinson and M. J. Garlepp (1991). "HLA antigen expression and malignant mesothelioma." American Journal of Respiratory Cell \& Molecular Biology 5(3): 213-220.

Christmas, T. I., L. S. Manning, M. J. Garlepp, A. W. Musk and B. W. Robinson (1993). "Effect of interferon-alpha 2a on malignant mesothelioma." J.Interferon.Res. 13(1): 9-12.

Colombo, M. P., G. Ferrari, A. Stoppacciaro, M. Parenza, M. Rodolfo, F. Mavilio and G. Parmiani (1991). "Granulocyte colony-stimulating factor gene transfer suppresses tumorigenicity of a murine adenocarcinoma in vivo." Journal of Experimental Medicine 173(4): 889-897.

Creaney, J., B. Mc Laren, S. Stevenson, A. Musk, N. de Klerk, B. W. Robinson and R. A. Lake (2001). "P53 autoantibodies in patients with Malignant Mesothelioma: Stability through disease progression." Br.J.Cancer 84: 52-56.

Davidson, J. A., A. W. Musk, B. R. Wood, S. Morey, M. Ilton, L. L. Yu, P. Drury, K. Shilkin and B. W. Robinson (1998). "Intralesional cytokine therapy in cancer: a pilot study of GM-CSF infusion in mesothelioma." J.Immunother. 21(5): 389-398.

Davis, M. R., L. S. Manning, D. Whitaker, M. J. Garlepp and B. W. Robinson (1992). "Establishment of a murine model of malignant mesothelioma." Int.J.Cancer 52(6): 881-886.

DeSilva, D. R., K. B. Urdahl and M. K. Jenkins (1991). "Clonal anergy is induced in vitro by T cell receptor occupancy in the absence of proliferation." Journal of Immunology 147(10): 3261-7.

Di Carlo, E., G. Forni, P. Lollini, M. P. Colombo, A. Modesti and P. Musiani (2001). "The intriguing role of polymorphonuclear neutrophils in antitumor reactions." Blood 97(2): 339-45.

Dranoff, G., E. Jaffee, A. Lazenby, P. Golumbek, H. Levitsky, K. Brose, V. Jackson, H. Hamada, D. Pardoll and R. C. Mulligan (1993). "Vaccination with irradiated tumor cells engineered to secrete murine granulocyte-macrophage colony-stimulating factor stimulates potent, specific, and longlasting anti-tumor immunity." Proceedings of the National Academy of Sciences of the United States of America 90(8): 3539-3543. 
Fearon, E. R., D. M. Pardoll, T. Itaya, P. Golumbek, H. I. Levitsky, J. W. Simons, H. Karasuyama, B. Vogelstein and P. Frost (1990). "Interleukin-2 production by tumor cells bypasses T helper function in the generation of an antitumor response." Cell 60(3): 397-403.

Fizazi, K., R. Caliandro, P. Soulie, A. Fandi, C. Daniel, A. Bedin, H. Doubre, J. Viala, J. Rodier, L. Trandafir, T. Le Chevalier, E. Cvitkovic, J. Armand and P. Ruffie (2000). "Combination raltitrexed (Tomudex(R))-oxaliplatin: a step forward in the struggle against mesothelioma? The Institut Gustave Roussy experience with chemotherapy and chemo-immunotherapy in mesothelioma." European Journal of Cancer 36(12): 1514-21.

Gansbacher, B., R. Bannerji, B. Daniels, K. Zier, K. Cronin and E. Gilboa (1990). "Retroviral vectormediated gamma-interferon gene transfer into tumor cells generates potent and long lasting antitumor immunity." Cancer Research 50(24): 7820-7825.

Garlepp, M., F. D, M. S, B.-O. H, D. M and R. BWS (1992). Mesothelioma: recent studies of growth regulation. Sourcebook on asbestos diseases :Asbestos medical research. P. G. New York, Garland Publishing.

Goey, S. H., A. M. Eggermont, C. J. Punt, R. Slingerland, J. W. Gratama, R. Oosterom, R. Oskam, R. L. Bolhuis and G. Stoter (1995). "Intrapleural administration of interleukin 2 in pleural mesothelioma: a phase I-II study." British Journal of Cancer 72(5): 1283-1288.

Golumbek, P. T., A. J. Lazenby, H. I. Levitsky, L. M. Jaffee, H. Karasuyama, M. Baker and D. M. Pardoll (1991). "Treatment of established renal cancer by tumor cells engineered to secrete interleukin-4." Science 254(5032): 713-716.

Grimm, E. A., A. Mazumder, H. Z. Zhang and S. A. Rosenberg (1982). "Lymphokine-activated killer cell phenomenon. Lysis of natural killer-resistant fresh solid tumor cells by interleukin 2-activated autologous human peripheral blood lymphocytes." Journal of Experimental Medicine 155(6): 1823-1841.

Hand, A., K. Pelin, K. Mattson and K. Linnainmaa (1995). "Interferon (IFN)-alpha and IFN-gamma in combination with methotrexate: in vitro sensitivity studies in four human mesothelioma cell lines." Anticancer Drugs 6(1): 77-82.

Hand, A. M., K. Husgafvel-Pursiainen, L. Tammilehto, K. Mattson and K. Linnainmaa (1991). "Malignant mesothelioma: the antiproliferative effect of cytokine combinations on three human mesothelioma cell lines." Cancer Lett. 58(3): 205-210.

Harrison, L. H., Jr, P. O. Schwarzenberger, P. S. Byrne, A. J. Marrogi, J. K. Kolls and K. E. McCarthy (2000). "Gene-modified PA1-STK cells home to tumor sites in patients with malignant pleural mesothelioma." Annals of Thoracic Surgery 2000 Aug;70(2):407-11 70(2): 407-411.

Haslam, P. L., A. Lukoszek, J. A. Merchant and M. Turner-Warwick (1978). "Lymphocyte responses to phytohaemagglutinin in patients with asbestosis and pleural mesothelioma." Clinical \& Experimental Immunology 31(2): 178-188.

Hellstrom, K. E. and I. Hellstrom (1974). "Lymphocyte-mediated cytotoxicity and blocking serum activity to tumor antigens." Advances in Immunology. 18: 209-77.

Imperiale, M. J., H. I. Pass and M. G. Sanda (2001). "Prospects for an SV40 vaccine." Seminars in Cancer Biology. 11(1): 81-5.

Jager, E., Y. T. Chen, J. W. Drijfhout, J. Karbach, M. Ringhoffer, D. Jager, M. Arand, H. Wada, Y. Noguchi, E. Stockert, L. J. Old and A. Knuth (1998). "Simultaneous humoral and cellular immune response against cancer-testis antigen NY-ESO-1: definition of human histocompatibility leukocyte antigen (HLA)-A2-binding peptide epitopes." Journal of Experimental Medicine 187(2): 265-270.

Kindzelskii, A. L. and H. R. Petty (1999). "Early membrane rupture events during neutrophil-mediated antibody-dependent tumor cell cytolysis." Journal of Immunology 162(6): 3188-92.

Leigh, R. A. and I. Webster (1982). "Lymphocytic infiltration of pleural mesothelioma and its significance for survival." South African Medical Journal 61(26): 1007-1009.

Leong, C. C., J. V. Marley, S. Loh, N. Milech, B. W. Robinson and M. J. Garlepp (1997). "Transfection of the gene for B7-1 but not B7-2 can induce immunity to murine malignant mesothelioma." Int.J.Cancer 71(3): 476-482.

Leong, C. C., J. V. Marley, S. Loh, B. W. Robinson and M. J. Garlepp (1997). "The induction of immune responses to murine malignant mesothelioma by IL-2 gene transfer." Immunol.Cell Biol. 75(4): 356-359. 
Lew, F., P. Tsang, J. F. Holland, N. Warner, I. J. Selikoff and J. G. Bekesi (1986). "High frequency of immune dysfunctions in asbestos workers and in patients with malignant mesothelioma." Journal of Clinical Immunology 6(3): 225-233.

Lissoni, P., S. Barni, A. Ardizzoia, F. Paolorossi, E. Tisi, S. Crispino and G. Tancini (1992). "Intracavitary administration of interleukin-2 as palliative therapy for neoplastic effusions." Tumori 78(2): 118120.

Manning, L. S., R. V. Bowman, S. B. Darby and B. W. Robinson (1989). "Lysis of human malignant mesothelioma cells by natural killer (NK) and lymphokine-activated killer (LAK) cells." Am.Rev.Respir.Dis. 139(6): 1369-1374.

Manning, L. S., M. R. Davis and B. W. Robinson (1991). "Asbestos fibres inhibit the in vitro activity of lymphokine-activated killer (LAK) cells from healthy individuals and patients with malignant mesothelioma." Clinical \& Experimental Immunology 83(1): 85-91.

Marzo, A. L., R. A. Lake, D. Lo, L. Sherman, A. McWilliam, D. Nelson, B. W. Robinson and B. Scott (1999a). "Tumor antigens are constitutively presented in the draining lymph nodes." Journal of Immunology. 162(10): 5838-45.

Marzo, A. L., R. A. Lake, B. W. Robinson and B. Scott (1999b). "T-cell receptor transgenic analysis of tumor-specific CD8 and CD4 responses in the eradication of solid tumors." Cancer Research. 59(5): 1071-9.

Matzinger, P. (1998). "An innate sense of danger." Seminars in Immunology 10(5): 399-415.

Medzhitov, R. and C. Janeway (2000). "The Toll receptor family and microbial recognition. [Review] [46 refs]." Trends in Microbiology 2000 Oct;8(10):452-6 8(10): 452-456.

Metintas, M., N. Ozdemir, I. Ucgun, O. Elbek, M. Kolsuz, S. Mutlu and S. Metintas (1999). "Cisplatin, mitomycin, and interferon-alpha2a combination chemoimmunotherapy in the treatment of diffuse malignant pleural mesothelioma." Chest 116(2): 391-398.

Mukherjee, S., T. Haenel, R. Himbeck, B. Scott, I. Ramshaw, R. A. Lake, G. Harnett, P. Phillips, S. Morey, D. Smith, J. A. Davidson, A. W. Musk and B. Robinson (2000). "Replication-restricted vaccinia as a cytokine gene therapy vector in cancer: persistent transgene expression despite antibody generation." Cancer Gene Therapy 2000 May;7(5):663-70 7(5): 663-670.

Mukherjee, S., D. Nelson, S. Loh, I. van Bruggen, L. J. Palmer, C. Leong, M. J. Garlepp and B. W. Robinson (2001). "The immune anti-tumor effects of GM-CSF and B7-1 gene transfection are enhanced by surgical debulking of tumor." Cancer Gene Therapy. 8(8): 580-8.

Naftzger, C., Y. Takechi, H. Kohda, I. Hara, S. Vijayasaradhi and A. N. Houghton (1996). "Immune response to a differentiation antigen induced by altered antigen: a study of tumor rejection and autoimmunity." Proceedings of the National Academy of Sciences of the United States of America 93(25): 14809-14.

Nowak, A. K., M. J. Byrne, R. Williamson, G. Ryan, A. Segal, D. Fielding, P. Mitchell, A. W. Musk and B. W. Robinson (2002). "A multicentre phase II study of cisplatin and gemcitabine for malignant mesothelioma." British Journal of Cancer 87(5): 491-6.

Nowak, A. K., B. W. Robinson and R. A. Lake (2002). "Gemcitabine exerts a selective effect on the humoral immune response: implications for combination chemo-immunotherapy." Cancer Research 62(8): 2353-8.

O'Brien, M. E., A. Saini, I. E. Smith, A. Webb, K. Gregory, R. Mendes, C. Ryan, K. Priest, K. V. Bromelow, R. D. Palmer, N. Tuckwell, D. A. Kennard and B. E. Souberbielle (2000). "A randomized phase II study of SRL172 (Mycobacterium vaccae) combined with chemotherapy in patients with advanced inoperable non-small-cell lung cancer and mesothelioma." British Journal of Cancer 2000 Oct;83(7):853-7 LM - 83(7): 853-857.

O'Reilly, E. M., D. H. Ilson, L. B. Saltz, R. Heelan, L. Martin and D. P. Kelsen (1999). "A phase II trial of interferon alpha-2a and carboplatin in patients with advanced malignant mesothelioma." Cancer Investigation 17(3): 195-200.

Pardoll, D. M. (1995). "Paracrine cytokine adjuvants in cancer immunotherapy. [Review] [63 refs]." Annual Review of Immunology 13: 399-415.

Pardoll, D. M. (1998). "Cancer vaccines. [Review] [128 refs]." Nature Medicine 4(5:Suppl): Suppl-31.

Pardoll, D. M. and S. L. Topalian (1998). "The role of CD4+ T cell responses in antitumor immunity." Current Opinion in Immunology. 10(5): 588-94. 
Parmiani, G., M. P. Colombo, C. Melani and F. Arienti (1997). "Cytokine gene transduction in the immunotherapy of cancer. [Review] [235 refs]." Advances in Pharmacology (New York) 40: 259307.

Porta, C., M. Danova, A. M. Orengo, S. Ferrini, M. Moroni, A. Gaggero, R. Libener, P. G. Betta, S. Ferrari, A. Procopio, L. Strizzi and L. Mutti (2000). "Interleukin-2 induces cell cycle perturbations leading to cell growth inhibition and death in malignant mesothelioma cells in vitro." Journal of Cellular Physiology 2000 Oct;185(1):126-34 185(1): 126-134.

Qin, Z., G. Richter, T. Schuler, S. Ibe, X. Cao and T. Blankenstein (1998). "B cells inhibit induction of T cell-dependent tumor immunity." Nature Medicine. 4(5): 627-30.

Robinson, B. (1989). "Asbestos and cancer: human natural killer cell activity is suppressed by asbestos fibres but can be restored by recombinant interleukin2." Am Rev Respir Dis 139: 897-901.

Robinson, B., R. Bowman, L. Manning, A. Musk and G. Van Hazel (1993). "Interleukin2 and lymphokine activated killer cells in malignant mesothelioma." Europ Resp Rev 3(11): 220-222.

Robinson, B. W., R. A. Lake, D. J. Nelson, B. A. Scott and A. L. Marzo (1999). "Cross-presentation of tumour antigens: evaluation of threshold, duration, distribution and regulation." Immunology \& Cell Biology. 77(6): 552-8.

Robinson, B. W., C. Robinson and R. A. Lake (2001). "Localized spontaneous mesothelioma regression possible immunological mechanism." Lung Cancer.

Robinson, C., M. Callow, S. Stevenson, B. Scott, B. W. Robinson and R. A. Lake (2000). "Serologic responses in patients with malignant mesothelioma: evidence for both public and private specificities." American Journal of Respiratory Cell \& Molecular Biology 2000 May;22(5):550-6 22(5): 550-556.

Schwarzenberger, P., L. Harrison, A. Weinacker, A. Marrogi, P. Byrne, R. Ramesh, C. Theodossiou, R. Gaumer, W. Summer, S. M. Freeman and J. K. Kolls (1998). "The treatment of malignant mesothelioma with a gene modified cancer cell line: a phase I study." Human Gene Therapy 9(17): 2641-2649.

Schwarzenberger, P., D. Lei, S. M. Freeman, P. Ye, A. Weinacker, C. Theodossiou, W. Summer and J. K. Kolls (1998). "Antitumor activity with the HSV-tk-gene-modified cell line PA-1-STK in malignant mesothelioma." American Journal of Respiratory Cell \& Molecular Biology 19(2): 333337.

Sigalotti, L., S. Coral, M. Altomonte, L. Natali, G. Gaudino, P. Cacciotti, R. Libener, F. Colizzi, G. Vianale, F. Martini, M. Tognon, A. Jungbluth, J. Cebon, E. Maraskovsky, L. Mutti and M. Maio (2002). "Cancer testis antigens expression in mesothelioma: role of DNA methylation and bioimmunotherapeutic implications." British Journal of Cancer 86(6): 979-82.

Simons, J. W., B. Mikhak, J. F. Chang, A. M. DeMarzo, M. A. Carducci, M. Lim, C. E. Weber, A. A. Baccala, M. A. Goemann, S. M. Clift, D. G. Ando, H. I. Levitsky, L. K. Cohen, M. G. Sanda, R. C. Mulligan, A. W. Partin, H. B. Carter, S. Piantadosi, F. F. Marshall and W. G. Nelson (1999). "Induction of immunity to prostate cancer antigens: results of a clinical trial of vaccination with irradiated autologous prostate tumor cells engineered to secrete granulocyte-macrophage colonystimulating factor using ex vivo gene transfer." Cancer Research 59(20): 5160-8.

Sklarin, N. T., A. P. Chahinian, E. J. Feuer, L. A. Lahman, L. Szrajer and J. F. Holland (1988). "Augmentation of activity of cis-diamminedichloroplatinum(II) and mitomycin C by interferon in human malignant mesothelioma xenografts in nude mice." Cancer Res. 48(1): 64-67.

Smith, J. W., W. J. Urba, J. W. Clark, D. L. Longo, M. Farrell, S. P. Creekmore, K. C. Conlon, H. Jaffe and R. G. Steis (1991). "Phase I evaluation of recombinant tumor necrosis factor given in combination with recombinant interferon-gamma." Journal of Immunotherapy 10(5): 355-362.

Soulie, P., P. Ruffie, L. Trandafir, I. Monnet, A. Tardivon, P. Terrier, E. Cvitkovic, C. T. Le and J. P. Armand (1996). "Combined systemic chemoimmunotherapy in advanced diffuse malignant mesothelioma. Report of a phase I-II study of weekly cisplatin/interferon alfa-2a." J.Clin.Oncol. 14(3): 878-885.

Suzuki, Y., A. P. Chahinian and T. Ohnuma (1987). "Comparative studies of human malignant mesothelioma in vivo, in xenografts in nude mice, and in vitro. Cell origin of malignant mesothelioma." Cancer 60(3): 334-344.

Tepper, R. I., R. L. Coffman and P. Leder (1992). "An eosinophil-dependent mechanism for the antitumor effect of interleukin-4." Science 257(5069): 548-551. 
Tepper, R. I., P. K. Pattengale and P. Leder (1989). "Murine interleukin-4 displays potent anti-tumor activity in vivo." Cell 57(3): 503-512.

Trandafir, L., P. Ruffie, C. Borel, I. Monnet, P. Soulie, D. Adams, E. Cvitkovic and J. P. Armand (1997). "Higher doses of alpha-interferon do not increase the activity of the weekly cisplatin-interferon combination in advanced malignant mesothelioma." Eur.J.Cancer 33(11): 1900-1902.

Upham, J. W., A. W. Musk, H. G. van, M. Byrne and B. W. Robinson (1993). "Interferon alpha and doxorubicin in malignant mesothelioma: a phase II study." Aust.N.Z.J.Med. 23(6): 683-687.

Vogelzang, N. J., J. Rusthoven, P. Paoletti, C. Denham, E. Kaukel, P. Ruffie, U. Gatzmeier, M. J. Boyer, S. Emri and C. Niyikiza (2002). Phase III single-blinded study of pemetrexed + cisplatin vs. cisplatin alone in chemonaive patients with malignant pleural mesothelioma. Proc. ASCO. Abstr. 5.

Von Hoff, D., B. Metch, J. G. Lucas, S. P. Balcerzak, S. M. Grunberg and S. E. Rivkin (1990). "Phase II evaluation of recombinant interferon-beta (IFN-beta ser) in patients with diffuse mesothelioma: a Southwest Oncology Group study." Journal of Interferon Research 10(5): 531-534.

Von Hoff, D. D. and A. M. Huong (1988). "Effect of recombinant interferon-beta ser on primary human tumor colony-forming units." Journal of Interferon Research 8(6): 813-820.

Vonderheide, R. H., J. P. Dutcher, J. E. Anderson, S. G. Eckhardt, K. F. Stephans, B. Razvillas, S. Garl, M. D. Butine, V. P. Perry, R. J. Armitage, R. Ghalie, D. A. Caron and J. G. Gribben (2001). "Phase I study of recombinant human CD40 ligand in cancer patients." Journal of Clinical Oncology 19(13): 3280-7.

Webster, I., J. W. Cochrane and K. R. Burkhardt (1982). "Immunotherapy with BCG vaccine in 30 cases of mesothelioma." South African Medical Journal 61(8): 277-278. 